Georgian Mathematical Journal

Volume 12 (2005), Number 2, 261-272

\title{
DISTURBANCE DUE TO A TIME HARMONIC SOURCE IN ORTHOTROPIC MICROPOLAR VISCOELASTIC MEDIUM
}

\author{
RAJNEESH KUMAR AND SUMAN CHOUDHARY
}

\begin{abstract}
The present study is concerned with the plane strain problem in homogeneous orthotropic micropolar viscoelastic solid. The disturbance due to the time harmonic concentrated source is investigated by employing the eigenvalue approach. Integral transforms have been inverted by a numerical technique to obtain the components of displacement, force stress and couple stress in the physical domain. The results obtained are given and illustrated graphically.
\end{abstract}

2000 Mathematics Subject Classification: 74F99, 74Q10, 74H15.

Key words and phrases: Micropolar, orthotropic, viscoelastic, eigenvalue and Fourier transforms.

\section{INTRODUCTION}

The anelastic behaviour of the Earth's material plays an important role in changing the characteristics of seismic waves, in defining seismic source functions [1] and in determining the internal structure of the Earth. The general theory of viscoelasticity describes the linear behaviour of both elastic and anelastic materials and provides a basis for describing the attenuation of seismic waves due to anelasticity.

Eringen [2] extended the theory of micropolar elasticity to obtain a linear constitutive theory for a micropolar material possessing internal friction. A problem on micropolar viscoelastic waves was discussed by McCarthy and Eringen [15]. They considered the propagation conditions and growth equations governing the propagation of waves in a micropolar viscoelastic medium. Manole [13] established uniqueness theorems in the theory of linear viscoleasticity and in the theory of micropolar linear viscoelasticity by using the Laplace transform technique.

Manole [14] presented variational theorems in the linear micropolar viscoelastic solid. Gale [3] studied Saint-Venant's problem in micropolar viscoelasticity. Dynamical problems of micropolar viscoelasticity were discussed by Kumar and Choudhary [8].

In many engineering problems, including the response of soils, geological materials and composites, the assumptions of isotropic behavior may not take into account some significant features of the continuum response. The formulation and solution of anisotropic problems is far more difficult and cumbersome than their isotropic counterparts. In the last years the elastodynamic response of an anisotropic continuum received attention of a number of researchers. In 
particular, transversely isotropic and orthotropic materials which may not be distinguished from each other in plane strain and plane stress cases, have been more regularly studied. Iesan ([5], [6], [7]) analyzed the static problems of plane micropolar strain of a homogeneous and orthotropic elastic solid, torsion problems of homogeneous and orthotropic cylinders in the linear theory of micropolar elasticity and bending of orthotropic micropolar elastic beams by terminals couple. Nakamura et al. [16] developed finite element method for orthotropic micropolar elasticity. Mechanical sources in orthotrpoic micropolar elastic medium discussed by Kumar and Choudhary [9].

Most of the problems studied so far, in micropolar elasticity, involve the use of potential functions. However the use of the eigenvalue approach has an advantage of finding solutions of equations in the coupled form directly in matrix terms, where as the potential function approach requires decoupling of equations. Yet, the eigenvalue approach has not been applied in a micropolar orthotropic medium. Mahalanabis and Manna [11] applied eigenvalue approach to linear micropolar elasticity by writing basic equations of linear micropolar elasticity in the form of a matrix differential equation. Mahalanabis and Manna [12] discussed the problem of linear micropolar thermoelasticity by using the eigenvalue approach. Recently Kumar et al. [10] have applied the eigenvalue approach to a micropolar elastic medium due to impulsive force at origin.

In the present work we study the response of a homogeneous orthotropic micropolar viscoelastic medium to the time harmonic source using eigenvalue approach and applying the integral transform technique.

\section{Problem Formulation}

We consider a homogeneous and orthotropic micropolar viscoelastic medium of infinite extent with Cartesian co-ordinate system $(x, y, z)$. To analyze internal displacements and stresses of the medium with time harmonic concentrated load, the continuum is divided into two half-spaces defined by

i. $I|x|+|z|<\infty, \quad-\infty<y \leq 0$,

ii. $I I|x|+|z|<\infty, \quad 0 \leq y<\infty$.

A time harmonic source is assumed to be acting on the interface of two halfspaces $(y=0)$. We apply an arbitrary load $F_{o}(x, t)=F_{o}(x) e^{\iota \omega t}$.

If we restrict our analysis parallel to the $x y$-plane with displacement vector $\vec{u}=\left(u_{1}, u_{2}, 0\right)$ and microrotation vector $\vec{\phi}=\left(0,0, \phi_{3}\right)$, then the basic equations in the dynamic theory of plane strain of homogeneous and orthotropic micropolar viscoelastic solids in the absence of body forces and body couples, given by Eringen [2], can be written as:

$$
\begin{aligned}
t_{j i, j} & =\rho \frac{\partial^{2} u_{i}}{\partial t^{2}} \\
m_{i 3, i}+\epsilon_{i j 3} t_{i j} & =\rho j \frac{\partial^{2} \phi_{3}}{\partial t^{2}}
\end{aligned}
$$


The constitutive relations, given by Iesan [5], can be written as

$$
\begin{array}{cc}
t_{11}=A_{11} \epsilon_{11}+A_{12} \epsilon_{22}, & t_{12}=A_{77} \epsilon_{12}+A_{78} \epsilon_{21}, \\
t_{21}=A_{78} \epsilon_{12}+A_{88} \epsilon_{21}, & t_{22}=A_{12} \epsilon_{11}+A_{22} \epsilon_{22}, \\
m_{13}=B_{66} \phi_{3,1}, & m_{23}=B_{44} \phi_{3,2},
\end{array}
$$

where

$$
\begin{aligned}
\epsilon_{i j} & =u_{j, i}+\epsilon_{j i 3} \phi_{3}, \\
A_{i j} & =A_{i j}^{\prime}+A_{i j}^{\prime \prime} \frac{\partial}{\partial t}, \\
B_{i j} & =B_{i j}^{\prime}+B_{i j}^{\prime \prime} \frac{\partial}{\partial t}
\end{aligned}
$$

In these relations, we have used the following notations: $t_{i j}$ are force stress tensor components, $m_{i j}$ are couple stress tensor components, $\epsilon_{i j}$ are micropolar strain tensor components, $u_{i}$ are displacement vector components, $\phi_{3}$ are microrotation vector components, $\epsilon_{i j k}$ is permutation symbol, $A_{i j}^{\prime}, A_{i j}^{\prime \prime}, B_{i j}^{\prime}, B_{i j}^{\prime \prime}$ are characteristic constants of the material, $\rho$ is the density, and $j$ is microinertia.

From equations (1)-(4), we obtain the field plane strain equations for orthotropic micropolar solids in the form:

$$
\begin{aligned}
\left(A_{11} \frac{\partial^{2}}{\partial x^{2}}+A_{88} \frac{\partial^{2}}{\partial y^{2}}\right) u_{1}+\left(A_{12}+A_{78}\right) \frac{\partial^{2} u_{2}}{\partial x \partial y}-K_{1} \frac{\partial \phi_{3}}{\partial y} & =\rho \frac{\partial^{2} u_{1}}{\partial t^{2}} \\
\left(A_{12}+A_{78}\right) \frac{\partial^{2} u_{1}}{\partial x \partial y}+\left(A_{77} \frac{\partial^{2}}{\partial x^{2}}+A_{22} \frac{\partial^{2}}{\partial y^{2}}\right) u_{2}-K_{2} \frac{\partial \phi_{3}}{\partial x} & =\rho \frac{\partial^{2} u_{2}}{\partial t^{2}} \\
\left(B_{66} \frac{\partial^{2}}{\partial x^{2}}+B_{44} \frac{\partial^{2}}{\partial y^{2}}-\chi\right) \phi_{3}+K_{1} \frac{\partial u_{1}}{\partial y}+K_{2} \frac{\partial u_{2}}{\partial x} & =\rho j \frac{\partial^{2} \phi_{3}}{\partial t^{2}}
\end{aligned}
$$

where

$$
K_{1}=A_{78}-A_{88}, \quad K_{2}=A_{77}-A_{78}, \quad \chi=K_{2}-K_{1} .
$$

We assume that the time harmonic behavior as

$$
\begin{aligned}
u_{i}(x, y, t) & =u_{i}(x, y) e^{\imath \omega t}, ; i=1,2, \\
\phi_{3}(x, y, t) & =\phi_{3}(x, y) e^{\imath \omega t},
\end{aligned}
$$

and introduce the dimensionless quantities

$$
\begin{gathered}
x^{*}=\frac{x}{h}, \quad y^{*}=\frac{y}{h}, \quad u_{i}^{*}=\frac{u_{i}}{h}, \\
\phi_{3}^{*}=\frac{A_{11}}{K_{1}} \phi_{3}, \quad t_{i j}^{*}=\frac{t_{i j}}{A_{11}}, \quad t^{*}=\sqrt{\frac{A_{11}}{\rho h^{2}} t} \\
m_{i j}^{*}=\frac{h}{B_{66}} m_{i j}, \quad \omega^{* 2}=\frac{\rho h^{2}}{A_{11}} \omega^{2},
\end{gathered}
$$

where $h$ is the parameter of dimensions of length.

Using equations (6) and (7), the system of equations (5) can be reduced to (dropping the asterisks for convenience)

$$
\left(A_{11} \frac{\partial^{2}}{\partial x^{2}}+A_{88} \frac{\partial^{2}}{\partial y^{2}}\right) u_{1}+\left(A_{12}+A_{78}\right) \frac{\partial^{2} u_{2}}{\partial x \partial y}
$$




$$
\begin{aligned}
-\frac{K_{1}^{2}}{A_{11}} \frac{\partial \phi_{3}}{\partial y} & =-A_{11} \omega^{2} u_{1}, \\
\left(A_{12}+A_{78}\right) \frac{\partial^{2} u_{1}}{\partial x \partial y}+\left(A_{77} \frac{\partial^{2}}{\partial x^{2}}+A_{22} \frac{\partial^{2}}{\partial y^{2}}\right) u_{2} & \\
-\frac{K_{1} K_{2}}{A_{11}} \frac{\partial \phi_{3}}{\partial x} & =-A_{11} \omega^{2} u_{2}, \\
\left(B_{66} \frac{\partial^{2}}{\partial x^{2}}+B_{44} \frac{\partial^{2}}{\partial y^{2}}-h^{2} \chi\right)\left(\frac{K_{1}}{A_{11} h^{2}}\right) \phi_{3} & \\
+K_{1} \frac{\partial u_{1}}{\partial y}+K_{2} \frac{\partial u_{2}}{\partial x} & =-\frac{j K_{1}}{h^{2}} \omega^{2} \phi_{3} .
\end{aligned}
$$

Applying the Fourier transform w.r.t $x$ defined by

$$
\left\{\tilde{u}_{i}(\xi, y, t), \tilde{\phi}_{3}(\xi, y, t)\right\}=\int_{-\infty}^{\infty}\left\{u_{i}(x, y, t), \phi_{3}(x, y, t)\right\} e^{\iota \xi x} d x, \quad i=1,2,
$$

to equations (8)-(10), we obtain

$$
\begin{aligned}
& \tilde{u}_{1}^{\prime \prime}=Q_{11} \tilde{u}_{1}+Q_{15} \tilde{u}_{2}^{\prime}+Q_{16} \tilde{\phi}_{3}^{\prime}, \\
& \tilde{u}_{2}^{\prime \prime}=Q_{22} \tilde{u}_{2}+Q_{23} \tilde{\phi}_{3}+Q_{24} \tilde{u}_{1}^{\prime}, \\
& \tilde{\phi}_{3}^{\prime \prime}=Q_{32} \tilde{u}_{2}+Q_{33} \tilde{\phi}_{3}+Q_{34} \tilde{u}_{1}^{\prime} .
\end{aligned}
$$

where

$$
\begin{aligned}
& Q_{11}=\frac{A_{11}\left(\xi^{2}-\omega^{2}\right)}{A_{88}}, \quad Q_{15}=\frac{\iota \xi\left(A_{12}+A_{78}\right)}{A_{88}}, \quad Q_{16}=\frac{K_{1}^{2}}{A_{11} A_{88}}, \\
& Q_{22}=\frac{\left(\xi^{2} A_{77}-\omega^{2} A_{11}\right)}{A_{22}}, \quad Q_{23}=-\frac{\iota \xi K_{1} K_{2}}{A_{11} A_{22}}, \quad Q_{24}=\frac{\iota \xi\left(A_{12}+A_{78}\right)}{A_{22}}, \\
& Q_{32}=\frac{\iota \xi h^{2} K_{2} A_{11}}{B_{44} K_{1}}, \quad Q_{33}=\frac{\left(\xi^{2} B_{66}+\chi^{2} h^{2}\right)-j \omega^{2} A_{11}}{B_{44}}, \quad Q_{34}=-\frac{h^{2} A_{11}}{B_{44}} .
\end{aligned}
$$

The system of equations (12)-(14) can be rewritten as

$$
\frac{d}{d y} W(\xi, y, \omega)=A(\xi, \omega) W(\xi, y, \omega)
$$

where

$$
\begin{gathered}
W=\left[\begin{array}{c}
U \\
U^{\prime}
\end{array}\right], \quad A=\left[\begin{array}{rr}
O & I \\
A_{2} & A_{1}
\end{array}\right], \quad U=\left[\begin{array}{l}
\tilde{u}_{1} \\
\tilde{u}_{2} \\
\tilde{\phi}_{3}
\end{array}\right], \quad O=\left[\begin{array}{lrr}
0 & 0 & 0 \\
0 & 0 & 0 \\
0 & 0 & 0
\end{array}\right], \\
I=\left[\begin{array}{lll}
1 & 0 & 0 \\
0 & 1 & 0 \\
0 & 0 & 1
\end{array}\right], A_{1}=\left[\begin{array}{rrr}
0 & Q_{15} & Q_{16} \\
Q_{24} & 0 & 0 \\
Q_{34} & 0 & 0
\end{array}\right], A_{2}=\left[\begin{array}{rrr}
Q_{11} & 0 & 0 \\
0 & Q_{22} & Q_{23} \\
0 & Q_{32} & Q_{33}
\end{array}\right],
\end{gathered}
$$

To solve equation (15), we take

$$
W(\xi, y, \omega)=X(\xi, \omega) e^{q y}
$$


so that

$$
A(\xi, \omega) W(\xi, y, \omega)=q W(\xi, y, \omega)
$$

which leads to an eigenvalue problem. The characteristic equation corresponding to the matrix $A$ is given by

$$
\operatorname{det}[A-q I]=0,
$$

which, on expansion, implies

$$
q^{6}-\lambda_{1} q^{4}+\lambda_{2} q^{2}-\lambda_{3}=0
$$

where

$$
\begin{aligned}
\lambda_{1}= & Q_{15} Q_{24}+Q_{16} Q_{34}+Q_{11}+Q_{22}+Q_{33}, \\
\lambda_{2}= & Q_{15}\left(Q_{24} Q_{33}-Q_{23} Q_{34}\right)+Q_{16}\left(Q_{22} Q_{34}-Q_{24} Q_{32}\right) \\
& +Q_{11} Q_{22}+Q_{22} Q_{33}+Q_{11} Q_{33}-Q_{23} Q_{32}, \\
\lambda_{3}= & Q_{11}\left(Q_{22} Q_{33}-Q_{23} Q_{32}\right) .
\end{aligned}
$$

The roots of equation (16) are $\pm q_{i}, \quad i=1,2,3$.

The vector $X(\xi)$ corresponding to the eigenvalues $q_{i}$ can be determined by solving the homogeneous equation

$$
[A-q I] X(\xi, \omega)=0 .
$$

The set of eigenvectors $X_{i}(\xi, \omega),(i=1,2,3,4,5,6)$ can be written as

$$
X_{i}(\xi, \omega)=\left[\begin{array}{c}
X_{i 1}(\xi, \omega) \\
X_{i 2}(\xi, \omega)
\end{array}\right]
$$

where

$$
\begin{aligned}
X_{i 1}(\xi, \omega) & =\left[\begin{array}{c}
a_{i} q_{i} \\
b_{i} \\
1
\end{array}\right], \quad X_{i 2}(\xi, \omega)=\left[\begin{array}{c}
a_{i} q_{i}^{2} \\
b_{i} q_{i} \\
q_{i}
\end{array}\right] \\
X_{j 1}(\xi, \omega) & =\left[\begin{array}{c}
-a_{i} q_{i} \\
b_{i} \\
1
\end{array}\right], \quad X_{j 2}(\xi, \omega)=\left[\begin{array}{c}
a_{i} q_{i}^{2} \\
-b_{i} q_{i} \\
-q_{i}
\end{array}\right], \\
j=i+3, & q=-q_{i} ; \quad i=1,2,3, \\
a_{i} & =\left(q_{i}^{2} Q_{15}+Q_{16} Q_{32}-Q_{15} Q_{33}\right) / \Delta_{i}, \\
b_{i} & =\left[q_{i}^{4}-q_{i}^{2}\left(Q_{16} Q_{34}+Q_{11}+Q_{33}\right)+Q_{11} Q_{33}\right] / \Delta_{i}, \\
\Delta_{i} & =q_{i}^{2}\left(Q_{15} Q_{34}+Q_{32}\right)-Q_{32} Q_{11} .
\end{aligned}
$$

The solution of equation (15) is given by

$$
W(\xi, y, \omega)=\sum_{i=1}^{3}\left[B_{i} X_{i}(\xi, \omega) \exp \left(q_{i} y\right)+B_{i+3} X_{i+3}(\xi, \omega) \exp \left(-q_{i} y\right)\right],
$$

where, $B_{i}(i=1,2,3,4,5,6)$ are arbitrary constants. 
Due to the eigenvalue approach equation (17) represents a solution of the general problem in the plane strain case of micropolar orthotropic elasticity and therefore can be applied to a broad class of problems in the domain of Fourier transforms.

For the half-space $y \geq 0$ and $y \leq 0$ the roots $q_{1}, q_{2}, q_{3}$ are related such that the real part of $\left(q_{1}, q_{2}, q_{3}\right) \geq 0$. With this consideration, the radiation conditions at infinity are satisfied and $B_{1}, B_{2}, B_{3}$ tend to zero for the domain as $y \rightarrow \infty$ and $B_{4}, B_{5}, B_{6}$ tend to zero for the domain as $y \rightarrow-\infty$, respectively.

\section{Application}

We consider an infinite orthrotropic micropolar viscoelastic space in which the concentrated force $F_{o}(x, t)=F_{o} \delta(x) e^{\imath \omega t}$ acts in the direction of the $y$-axis at the origin of the Cartesian co-ordinate system. The boundary conditions at the interface of two half-spaces $(y=0)$ are given by

$$
\begin{aligned}
u_{1}\left(x, 0^{+}, t\right)-u_{1}\left(x, 0^{-}, t\right) & =0, \quad u_{2}\left(x, 0^{+}, t\right)-u_{2}\left(x, 0^{-}, t\right)=0, \\
\phi_{3}\left(x, 0^{+}, t\right)-\phi_{3}\left(x, 0^{-}, t\right) & =0, \quad m_{23}\left(x, 0^{+}, t\right)-m_{23}\left(x, 0^{-}, t\right)=0, \\
t_{21}\left(x, 0^{+}, t\right)-t_{21}\left(x, 0^{-}, t\right) & =0, \\
t_{22}\left(x, 0^{+}, t\right)-t_{22}\left(x, 0^{-}, t\right) & =-F_{o} \delta(x) e^{\iota \omega t} .
\end{aligned}
$$

Using equations (6) and (7) and then applying the Fourier transforms from equation (11) to the system of equations (18), we get

$$
\begin{aligned}
\tilde{u}_{1}\left(\xi, 0^{+}, \omega\right)-\tilde{u}_{1}\left(\xi, 0^{-}, \omega\right) & =0, \quad \tilde{u}_{2}\left(\xi, 0^{+}, \omega\right)-\tilde{u}_{2}\left(\xi, 0^{-}, \omega\right)=0, \\
\tilde{\phi}_{3}\left(\xi, 0^{+}, \omega\right)-\tilde{\phi}_{3}\left(\xi, 0^{-}, \omega\right) & =0, \quad \tilde{m}_{23}\left(\xi, 0^{+}, \omega\right)-\tilde{m}_{23}\left(\xi, 0^{-}, \omega\right)=0, \\
\tilde{t}_{21}\left(\xi, 0^{+}, \omega\right)-\tilde{t}_{21}\left(\xi, 0^{-}, \omega\right) & =0, \\
\tilde{t}_{22}\left(\xi, 0^{+}, \omega\right)-\tilde{t}_{22}\left(\xi, 0^{-}, \omega\right) & =-F_{o} .
\end{aligned}
$$

The transformed displacement, microrotation and stress components are given for $y \geq 0$ as

$$
\begin{aligned}
\tilde{u}_{1}(\xi, y, t) & =-\left(a_{1} q_{1} B_{4} e^{-q_{1} y}+a_{2} q_{2} B_{5} e^{-q_{2} y}+a_{3} q_{3} B_{6} e^{-q_{3} y}\right) e^{\imath \omega t}, \\
\tilde{u}_{2}(\xi, y, t) & =\left(b_{1} B_{4} e^{-q_{1} y}+b_{2} B_{5} e^{-q_{2} y}+b_{3} B_{6} e^{-q_{3} y}\right) e^{\imath \omega t} \\
\tilde{\phi}_{2}(\xi, y, t) & =\left(B_{4} e^{-q_{1} y}+B_{5} e^{-q_{2} y}+B_{6} e^{-q_{3} y}\right) e^{\imath \omega t} \\
\tilde{m}_{23}(\xi, y, t) & =-\frac{B_{44} K_{1}}{B_{66} A_{11}}\left(q_{1} B_{4} e^{-q_{1} y}+q_{2} B_{5} e^{-q_{2} y}+q_{3} B_{6} e^{-q_{3} y}\right) e^{\iota \omega t}, \\
\tilde{t}_{21}(\xi, y, t) & =\left(M_{1} B_{4} e^{-q_{1} y}+M_{2} B_{5} e^{-q_{2} y}+M_{3} B_{6} e^{-q_{3} y}\right) e^{\imath \omega t} \\
\tilde{t}_{22}(\xi, y, t) & =-\left(N_{1} B_{4} e^{-q_{1} y}+N_{2} B_{5} e^{-q_{2} y}+N_{3} B_{6} e^{-q_{3} y}\right) e^{\imath \omega t},
\end{aligned}
$$

and for $y \leq 0$ as

$$
\begin{aligned}
& \tilde{u}_{1}(\xi, y, t)=\left(a_{1} q_{1} B_{1} e^{q_{1} y}+a_{2} q_{2} B_{2} e^{q_{2} y}+a_{3} q_{3} B_{3} e^{q_{3} y}\right) e^{\iota \omega t} \\
& \tilde{u}_{2}(\xi, y, t)=\left(b_{1} B_{1} e^{q_{1} y}+b_{2} B_{2} e^{q_{2} y}+b_{3} B_{3} e^{q_{3} y}\right) e^{\iota \omega t} \\
& \tilde{\phi}_{2}(\xi, y, t)=\left(B_{1} e^{q_{1} y}+B_{2} e^{q_{2} y}+B_{3} e^{q_{3} y}\right) e^{\iota \omega t}
\end{aligned}
$$




$$
\begin{aligned}
\tilde{m}_{23}(\xi, y, t) & =\frac{B_{44} K_{1}}{B_{66} A_{11}}\left(q_{1} B_{1} e^{q_{1} y}+q_{2} B_{2} e^{q_{2} y}+q_{3} B_{3} e^{q_{3} y}\right) e^{\imath \omega t}, \\
\tilde{t}_{21}(\xi, y, t) & =\left(M_{1} B_{1} e^{q_{1} y}+M_{2} B_{2} e^{q_{2} y}+M_{3} B_{3} e^{q_{3} y}\right) e^{\imath \omega t}, \\
\tilde{t}_{22}(\xi, y, t) & =\left(N_{1} B_{1} e^{q_{1} y}+N_{2} B_{2} e^{q_{2} y}+N_{3} B_{3} e^{q_{3} y}\right) e^{\iota \omega t} .
\end{aligned}
$$

where

$$
\begin{aligned}
M_{i} & =\left(-\iota \xi A_{78} b_{i}+A_{88} a_{i} q_{i}^{2}\right) / A_{11}+K_{1}\left(A_{88}-A_{78}\right) / A_{11}^{2} \\
N_{i} & =\left(A_{22} b_{i}-\iota \xi A_{12} a_{i}\right) q_{i} / A_{11}
\end{aligned}
$$

and

$$
\begin{aligned}
& B_{1}=B_{4}=\frac{F_{o}\left(a_{2}-a_{3}\right) q_{2} q_{3}}{\Delta}, \\
& B_{2}=B_{5}=\frac{F_{o}\left(a_{3}-a_{1}\right) q_{1} q_{3}}{\Delta}, \\
& B_{3}=B_{6}=\frac{F_{o}\left(a_{1}-a_{2}\right) q_{1} q_{2}}{\Delta},
\end{aligned}
$$

where

$$
\Delta=2\left[q_{1} q_{2} N_{3}\left(a_{1}-a_{2}\right)+q_{2} q_{3} N_{1}\left(a_{2}-a_{3}\right)+q_{1} q_{3} N_{2}\left(a_{3}-a_{1}\right)\right] .
$$

Thus, by equation (17), the functions $\tilde{u}_{1}, \tilde{u}_{2}, \tilde{\phi}_{3}, \tilde{m}_{23}, \tilde{t}_{21}$ and $\tilde{t}_{22}$ can be determined in the transformed domain and enable us to find displacements, microrotations and stresses.

Particular case: Taking

$$
\begin{aligned}
A_{11}=A_{22}=\lambda+2 \mu+K, & A_{77}=A_{88}=\mu+K, \\
A_{12}=\lambda, A_{78}=\mu, & B_{44}=B_{66}=\gamma,
\end{aligned}
$$

with

$$
-K_{1}=K_{2}=\chi / 2=K,
$$

we obtain the corresponding expressions in the micropolar theory of elasticity.

\section{INVERSION OF TRANSFORMS}

The transformed displacements and stresses are the functions of $y$, the parameters of Fourier transforms $\xi$, and hence are of the form $\tilde{f}(\xi, y, t)$. To get the function $f(x, y, t)$ in the physical domain, first we invert the Fourier transform using

$$
\begin{aligned}
f(x, y, t) & =\frac{1}{2 \pi} \int_{-\infty}^{\infty} e^{-\iota \xi x} \tilde{f}(\xi, y, t) d \xi \\
& =\frac{1}{\pi} \int_{0}^{\infty}\left\{\cos (\xi x) f_{e}-\iota \sin (\xi x) f_{o}\right\} d \xi
\end{aligned}
$$

where $f_{e}$ and $f_{o}$ are the even and the odd part of the function $\tilde{f}(\xi, y, t)$ respectively. Thus expression (19) gives us the function $f(x, y, t)$. 
The last step is to evaluate the integral in equation (19) by the method due to Press et al. [17], which involves the use of Romberg's integration with adaptive step size. In applying this method we also use the corresponding results obtained by a successive refinement of the extended trapezoidal rule followed by their extrapolation to the limit as the step size tends to zero.

\section{Numerical Results and Discussion}

For numerical computations, we take the following values of relevant parameters for an orthotropic micropolar solid:

$$
\begin{array}{cl}
A_{11}^{\prime}=13.97 \times 10^{10} \text { dyne } / \mathrm{cm}^{2}, & A_{22}^{\prime}=13.75 \times 10^{10} \text { dyne } / \mathrm{cm}^{2}, \\
A_{77}^{\prime}=3.0 \times 10^{10} \text { dyne } / \mathrm{cm}^{2}, & A_{88}^{\prime}=3.2 \times 10^{10} \text { dyne } / \mathrm{cm}^{2}, \\
A_{12}^{\prime}=8.13 \times 10^{10} \text { dyne } / \mathrm{cm}^{2}, & A_{78}^{\prime}=2.2 \times 10^{10} \text { dyne } / \mathrm{cm}^{2}, \\
B_{44}^{\prime}=0.056 \times 10^{10} \text { dyne }, & B_{66}^{\prime}=0.057 \times 10^{10} \text { dyne }, \\
h=0.01 \mathrm{~cm} . &
\end{array}
$$

For a particular model of the orthotrpoic micropolar viscoelastic solid the corresponding parameters are expressed as

$$
\begin{array}{ll}
A_{11}=A_{11}^{\prime}\left(1+\iota Q_{1}^{-1}\right), & A_{22}=A_{22}^{\prime}\left(1+\iota Q_{2}^{-1}\right), \\
A_{77}=A_{77}^{\prime}\left(1+\iota Q_{3}^{-1}\right), & A_{88}=A_{88}^{\prime}\left(1+\iota Q_{4}^{-1}\right), \\
A_{12}=A_{12}^{\prime}\left(1+\iota Q_{5}^{-1}\right), & A_{78}=A_{78}^{\prime}\left(1+\iota Q_{6}^{-1}\right), \\
B_{44}=B_{44}^{\prime}\left(1+\iota Q_{7}^{-1}\right), & B_{66}=B_{66}^{\prime}\left(1+\iota Q_{8}^{-1}\right) .
\end{array}
$$

where

$$
\begin{array}{llll}
Q_{1}^{-1}=1.0, & Q_{2}^{-1}=0.5, & Q_{3}^{-1}=0.1, & Q_{4}^{-1}=1.5 \\
Q_{5}^{-1}=1.5, & Q_{6}^{-1}=1.0, & Q_{7}^{-1}=0.5, & Q_{8}^{-1}=1.0 .
\end{array}
$$

For the comparison with a micropolar isotropic solid, following Gauthier [4], we take the following values for the case of the corresponding parameters Aluminum epoxy composite:

$$
\begin{aligned}
\rho=2.19 \mathrm{gm} / \mathrm{cm}^{3}, & \lambda=7.59 \times 10^{10} \mathrm{dyne} / \mathrm{cm}^{2}, \\
\mu=1.89 \times 10^{10} \text { dyne } / \mathrm{cm}^{2}, & K=0.0149 \times 10^{10} \mathrm{dyne} / \mathrm{cm}^{2}, \\
\gamma=0.0268 \times 10^{10} \text { dyne }, & j=0.00196 \mathrm{~cm}^{2} .
\end{aligned}
$$

The comparison of the values of the dimensionless displacement component $U_{2}\left[=u_{2} / F_{o}\right]$, normal force stress $T_{22}\left[=t_{22} / F_{o}\right]$ and couple stress $M_{23}\left[=m_{23} / F_{o}\right]$, for micropolar orthotropic viscoelastic solid (MOVS), a micropolar isotopic viscoelastic solid (MIVS), a micropolar orthotropic solid (MOS), a micropolar isotopic solid (MIS) have been studied. Computations were carried out for two values of dimensionless frequency $\omega=0.25$ and $\omega=0.50$ for $t=1.0$ at $y=1.0$ in the range $0 \leq x \leq 10$. The solid lines either without or with the center symbol represent variations for the case of MOVS whereas the small dashed lines with or without center symbol represent the variations for case of MOS, medium dashed lines with or without the center symbol represent variations 
for the case of MIVS and the large dashed lines with or without the center symbol represent variations for the case of MIS. The curves without the center symbol corresponds to the case for $\omega=0.25$ whereas those with center symbol correspond to the case for $\omega=0.50$.

Figure 1 shows variations of the displacement component $U_{2}$ with distance $x$. For all the four cases of MOVS, MOS, MIVS and MIS and for both frequencies, initially the values of $U_{2}$ decrease sharply and as the frequency increases from 0.25 to 0.50 , the values of $U_{2}$ decrease in the range $0 \leq x \leq 2$. For both frequencies, the displacement component values are greater for the case of MOVS and smaller for the case of MIS. The behaviour of variations of $U_{2}$ for all the cases are oscillatory.

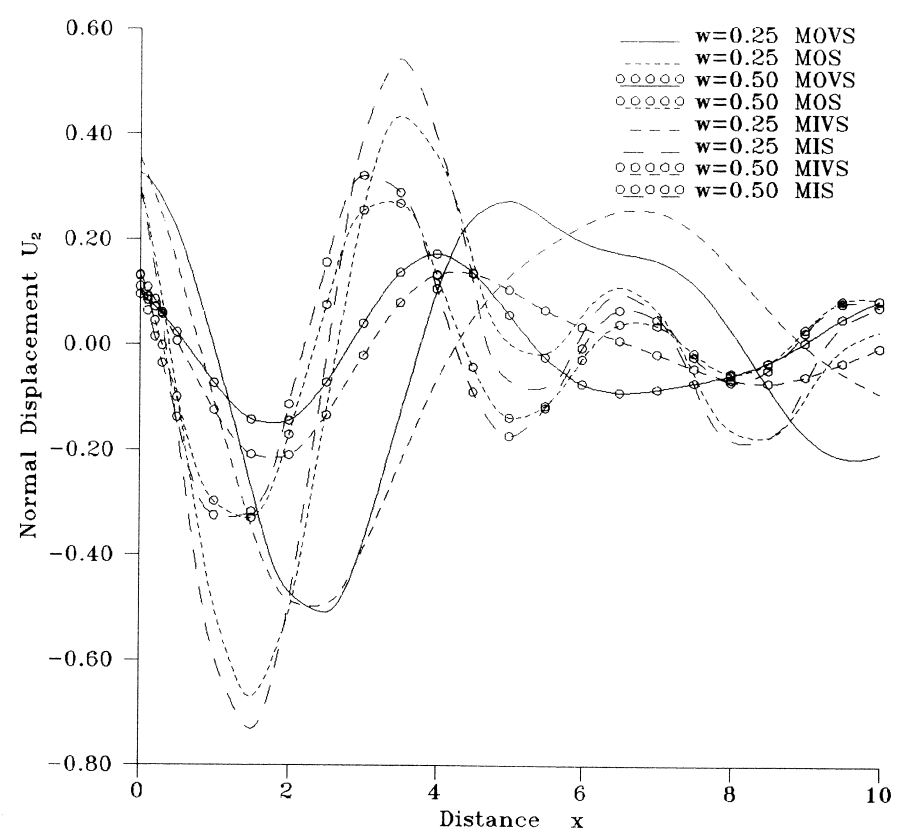

Fig. 1 Variations of Normal Displacement $U_{2}\left(=\mathbf{u}_{2} / F_{\mathrm{o}}\right)$ with Distance $x$.

Figure 2 shows variations of the normal force stress $T_{22}$ with $x$. Initially, the stress values are greater for the cases of MOS and MIS than those for MOVS and MIVS and the behaviour of their variations is opposite to each other. For the case of MOVS and MIVS and for both frequencies the values of $T_{22}$ increase in the initial ranges whereas for the case of MOS and MIS and for both the frequencies the values of $T_{22}$ increase in the initial ranges whereas for the case of MOS a nd MIS and both frequencies the values of $T_{22}$ decrease in the initial ranges. 


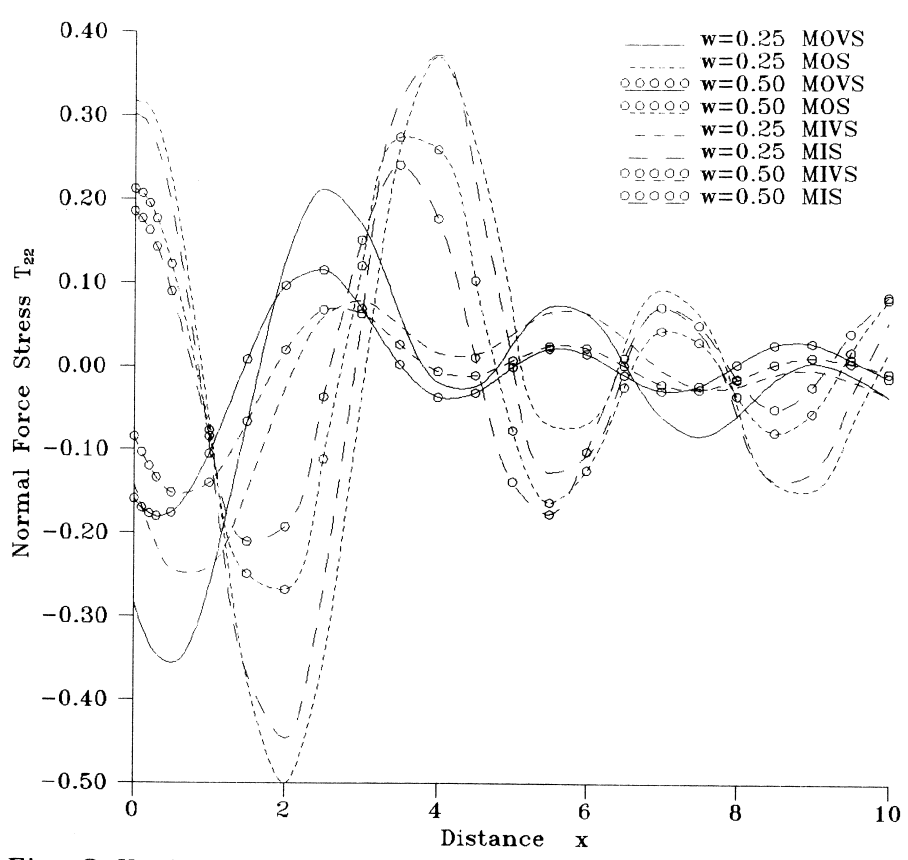

Fig. 2 Variations of Normal Force Stress $T_{22}\left(=t_{22} / F_{0}\right)$ with Distance $\mathrm{x}$.

Figure 3 shows variations of the couple stress $M_{23}$ with $x$. For all the four cases and for both frequencies the couple stress values initially increase and then start oscillating. the values of $M_{23}$ are greater for the case of MOS and smaller for the case of MOVS very near to the point of application of source.

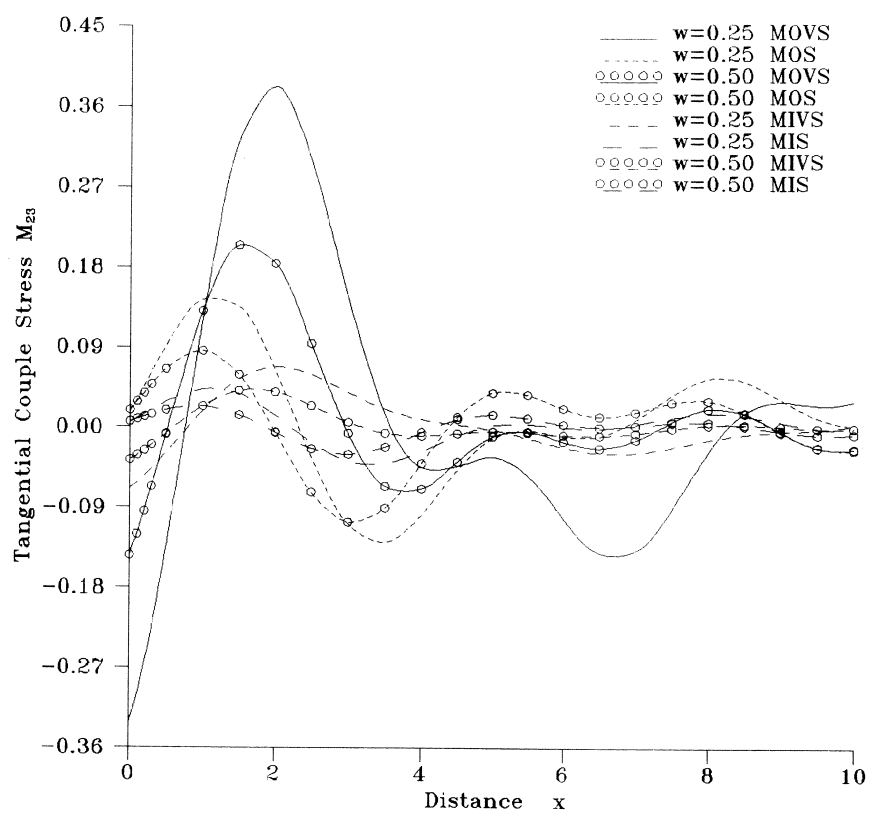

Fig. 3 Variations of Tangential Couple Stress $\mathrm{M}_{23}\left(=\mathrm{m}_{23} / \mathrm{F}_{\mathrm{o}}\right)$ with Distance $\mathrm{x}$. 
Conclusion: A significant anisotropy and viscoelastic effect on displacement component, force stress and couple stress is obtained for different frequencies. Near the point of application of load, the frequency plays a major role in all components.

\section{REFERENCES}

1. J. N. Brune, Tectonic stress and the spectra of seismic shear waves from earthquake. $J$. Geophys. Res. 751970, 4997-5009.

2. A. C. ERIngen, Linear theory of micropolar viscoelasticity. Internat. J. Engrg. Sci. 5(1967), 191-210.

3. C. Gale, On Saint-Venant's problem in micropolar viscoelasticity. An. Stiinţ. Univ. Al. I. Cuza Iaşi. Mat. (N.S.) 46(2000) 131-148.

4. R. D. Gauthier, In experimental investigations on micropolar media. Mechanics of Micropolar media (eds. O. Brulin and R. K. T. Hsieh), World Scientific, Singapore, 1982.

5. D. IEŞAn, The plane micropolar strain of orthotropic elastic solids. Arch. Mech. (Arch. Mech. Stos.) 25(1973), 547-561.

6. D. Ieşan, Torsion of anisotropic micropolar elastic cylinders. Z. Angew. Math. Mech. 54(1974), 773-779.

7. D. IEşAn, Bending of orthotropic micropolar elastic beams by terminal couples. An. Ştiinţ. Univ. Al. I. Cuza Iaşi, n. Ser., Sect. Ia 20(1974), 411-418.

8. R. Kumar and S. Choudhary, Dynamical Problem of micropolar viscoelasticity. Proc. Indian Acad. Sci. (Earth Planet. Sci.) 110(2001), 215-223.

9. R. Kumar and S. Choudhary, Mechanical sources in orthotropic micropolar continua. Proc. Indian Acad. Sci. (Earth Planet. Sci.) 111(2002), 133-141.

10. R. Kumar, R. Singh, and T. K. Chadha, Eigen value approach to micropolar medium due to impulsive force at the origin. Indian J. Pure Appl. Math. 32(2001), 1127-1144.

11. R. K. Mahalanabis and J. Manna, Eigenvalue approach to linear micropolar elasticity. Indian J. Pure Appl. Math. 20(1989), No. 12, 1237-1250.

12. R. K. Mahalanabis and J. Manna, Eigenvalue approach to the problem of linear micropolar thermoelasticity. J. Indian Acad. Math. 19(1997), No. 1, 69-86.

13. D. Manole, Théorème d'unicité dans la théorie de la viscoélasticité linéaire avec microstructure en utilisant la transformation de Laplace. Rev. Roumaine Sci. Tech. Sér. Méc. Appl. 33(1988), No. 3, 209-214.

14. D. Manole, Variational theorems in linear theory of micropolar viscoelasticity. Bul. Inst. Politehn. Iaşi Secţ. I 38(42)(1992), No. 1-4, 75-83.

15. M. F. MCCARthy and A. C. Eringen, Micropolar viscoelastic waves. Internat. J. Engrg. Sci. 7(1969), 447-458.

16. S. Nakamura, R. Benedict, and R. Lakes, Finite elament method for orthotropic micropolar elasticity. Internat. J. Engng. Sci. 22(1984), 319-330.

17. W. H. Press, S. A. Teukolsky, W. T. Vellerlig, and B. P. Flannery, Numerical recipes in FORTRAN. 2nd ed. Cambridge University Press, Cambridge, 1986.

(Received 20.11.2002; revised 26.10.2004) 
Authors' address:

Mathematics Department

Kurukshetra University

Kurukshetra 136119

Haryana, India

E-mail: suman_dalal2002@yahoo.co.in 